\title{
CT Classification of Vacuum Phenomenon Morphology and its Utility in Predicting Lumbar Vertebral Instability
}

Noah Kelm ( $\square$ noerke0501@gmail.com )

Ascension Genesys Hospital https://orcid.org/0000-0001-9642-135X

Jennifer Hella

Ascension Genesys Hospital

John B Westfall

Michigan State University

Eric T Ballard

Michigan State University

Macksood A Aftab

Ascension Genesys Hospital

\section{Research article}

Keywords: Vacuum Phenomenon, Intervertebral Disc, Intervertebral Disc Degeneration, Lumbar Spinal Instability, Computed Tomography Flexion-Extension X-rays, Lumbar Spinal Stenosis, Lumbar fusion

Posted Date: August 21st, 2020

DOI: https://doi.org/10.21203/rs.3.rs-57328/v1

License: (c) (i) This work is licensed under a Creative Commons Attribution 4.0 International License. Read Full License 


\section{Abstract}

Background: Lumbar vacuum phenomenon (VP) within the intervertebral disc has been classified based on CT imaging. We compared same-patient sagittal CT images and dynamic flexion-extension x-rays to determine if there is a difference in the amount of vertebral instability present between three VP morphologies on CT.

Methods: Anterior subluxation measurements on x-ray were compared with same-segment VP on CT images from the same patient when both findings were present. VP were classified as spot, island, or linear. It was determined if there was a difference in the amount of anterior subluxation between the three morphologies. Secondary analysis looked at whether there was a difference in anterior subluxation between the three groups if patients had undergone a prior lumbar fusion surgery or not.

Results: There was no difference in anterior subluxation between the three groups on dynamic flexionextension $\mathrm{x}$-rays. There was also no difference between the three groups on flexion-extension $\mathrm{x}$-rays when patients were separated based on if they had received or not received a previous lumbar fusion surgery.

Conclusion: IVD VP morphology is not a useful indicator in determining vertebral instability preoperatively according to CT scan. Further fine-tuning of an IVD VP CT classification is needed to help radiologists and spine surgeons know when IVD VP presence is important.

\section{Background}

As intervertebral disc (IVD) degeneration progresses, vertebral instability may ensue. Traditionally, flexionextension x-ray images of the spine have been utilized by spine surgeons prior to surgery to determine if dynamic subluxation of adjacent vertebrae or disc height narrowing is present, which helps guide surgical treatment. For instance, the presence of dynamic subluxation pre-operatively may indicate that spinal decompression surgery for spinal stenosis or nerve root impingement may render the spine even more unstable and requires the additional need for fusion with rod and screw constructs or interbody devices. ${ }^{1}$

Adjacent vertebrae articulate via the facet joints, but also through the vertebral bodies themselves. Each vertebral segment consists of a cranial and caudal vertebral body and its IVD. These discs allow for flexibility of the vertebral column without the significant sacrifice of strength, as well as providing a shock-absorbing effect. ${ }^{2}$ The IVD consists of an outer annulus fibrosis (AF) made of 15-25 sheets or "lamellae" comprised of mostly collagen fibers that provides tensile strength, while the inner nucleus propulsus (NP) is comprised of proteoglycan molecules that absorb water, which allows the disc to resist compressive forces by dispersing hydraulic pressure throughout the entire disc when loaded. ${ }^{3}$ Aging results in the loss of proteoglycans, which causes the NP to shrink and become fibrotic. More stress is transmitted to the AF, causing it to thicken. As a result, the disc flattens, collagen fibers are lost in the inner $A F$, and clefts and crevices can form. ${ }^{2,4-5}$ The increasing size of these clefts and crevices can lead to the accumulation of gases within them. 
The vacuum phenomenon (VP) is a universally accepted term among surgeons and radiologists referring to gaseous lucencies seen in the vertebral bodies, IVD's, facet joints, and spinal canal on x-ray and CT imaging. ${ }^{6,7}$ While originally thought to be due to a variety of pathologies, it has been established in literature that lumbar spine VP is usually due to degenerative changes, and is useful for eliminating infectious, malignant, or traumatic spinal pathologies. ${ }^{8-10}$

Recently, CT imaging of VP has been classified based on morphologic characteristics and location within the IVD. This 2-part classification system has demonstrated that certain characteristics of IVD VP are associated with degenerative disc disease and lumbar canal stenosis on MRI, as well as disc height narrowing on x-ray imaging. ${ }^{3}$ With this study, we look to implement this new classification scheme in our institution on patients with both $\mathrm{CT}$ and dynamic flexion-extension $\mathrm{x}$-rays from the same time period to determine if VP morphology can predict vertebral instability prior to spine surgery. If a correlation is present, VP on lumbar CT imaging may become a useful radiologic tool for spine surgeons when analyzing different treatment options pre-operatively. Furthermore, we will compare CT images and flexion-extension x-rays on patients who have previously undergone lumbar fusion to see if VP can measure vertebral instability post-operatively. We will also compare our findings between those who have had previous fusion to those who have not had a previous fusion.

\section{Methods}

A retrospective study was conducted at a community teaching hospital. Institutional review board approval was obtained. Using our institution's electronic radiology database, all patients who had undergone a lumbar CT and lateral lumbar flexion-extension x-rays of the lumbar spine between January 1, 2017-October 31,2019 were considered for enrollment in our study. Males and females of all ethnic backgrounds and ages who had received both radiologic studies during the same time period were included in our study. Patients who had received prior lumbar surgery (i.e. vertebral augmentation, decompression, fusion with screws/rods or interbody device, or discectomy) prior to CT were included. If patients who had undergone surgery were included, then x-rays and CT's both had to take place following surgery for a valuable comparison. Exclusion criteria were patients who had imaging of pathologies other than degenerative changes including infectious, malignant, or traumatic findings.

Patient data were collected and managed using REDCap (Research Electronic Data Capture) electronic data capture tools hosted at Ascension Genesys Hospital. REDCap is a secure, web-based software platform designed to support data capture for research studies. The presence of IVD VP's were determined on CT images. Only VP located in discs between T12-S1 were considered. If VP was present at discs in multiple levels in the lumbar spine, or multiple VP's were present within the same disc, the largest measured VP was collected for our study in millimeters $(\mathrm{mm})$. Therefore, for the sake of simplification, there is a 1:1 ratio of VP's to patients in our study. Using one dimension of the Murata et al. CT VP classification system, spot was considered any circular or oval-shaped VP less than $2 \mathrm{~mm}$ in diameter, while island was circular or oval and greater than $2 \mathrm{~mm}$ in diameter. Linear VP was linear, serpentine, or geographical shaped VP (Fig. 1). Lateral flexion-extension radiographs of the lumbar spine 
were then looked at. Only the vertebral segments including the measured VP's were analyzed for dynamic subluxation (Fig. 2). At these levels, we also measured the anterior disc height (ADH) and posterior disc height (PDH). The ADH was measured as the distance between the most anterior point of the cranial and caudal endplates, while the PDH was the measurement between the most posterior points of the cranial and caudal endplates.

The primary question of this study is whether there is a significant difference between spot, island, and linear VP morphologies regarding anterior subluxation on flexion-extension x-rays. Based on the sheer size of the island and linear VP being greater than spot VP, we hypothesize that these two morphologies will have a larger amount of dynamic subluxation on flexion-extension radiographs than spot VP. Subanalysis questions included whether there was a difference between $\mathrm{ADH}$ and $\mathrm{PDH}$ within the three morphologic groups on either flexion or extension x-ray views. In order to eliminate a potential confounding variable, we stratified the groups based on whether the patients had received a prior lumbar fusion surgery or not, and within these two groups, we compared the three different morphologies based on the amount of dynamic subluxation. Lastly, we compared the size of the VP's of those who had never had a previous lumbar surgery with those who have had a previous lumbar surgery with the hypothesis that lumbar fusion would decrease VP size.

\section{Statistical Analysis}

Descriptive statistics included frequencies and percentages. Student's t-Test and One-way ANOVA were used to compare groups depending on whether we were comparing between two or three means, respectively. Significance was determined as a p-value $\leq 0.05$ at a $95 \%$ confidence level.

\section{Results}

In this study, 114 patients between the ages of 32 and 88 years old (mean age: 63.3 years, SD: 13.1) were assessed. Of these patients, $80(70.2 \%)$ did not have previous surgery, while $34(29.8 \%)$ did have previous surgery. Of the 114 patients included, 88 of the patients had measurable VP in at least one level. Of these patients, 59 had no previous surgery, while 29 had previous surgery.

Of the CT images with VP present, $8 \%$ were spot $(n=7$, no surgery $=4$, previous surgery $=3), 70.5 \%$ were island $(n=62$, no surgery $=42$, previous surgery $=20)$, and $21.6 \%$ were linear $(n=19$, no surgery $=13$, previous surgery $=6$ ).

\section{Dynamic subluxation comparison between spot, island, and linear VP}

When comparing the amount of dynamic subluxation on flexion and extension x-rays between spot, island, and linear morphologies, there was no significant difference in our study (Table 1). When patients were stratified based on no surgery vs. previous surgery patients, there was no significant difference in 
flexion or extension subluxation between the three morphologies within the sample of patients with no surgery or within those with previous surgery (Table 2).

When comparing flexion to extension $x$-rays within the same patients, there was no subluxation significance within the spot group $(p=0.56)$, island group $(p=0.45)$, or linear group $(p=0.77)$.

Table 1

Dynamic subluxation comparison between spot, island and linear VP

\begin{tabular}{|llll|}
\hline & N & Mean (Standard Deviation) & P-value \\
\hline Flexion Subluxation & 7 & $4.9(5.5)$ & 0.29 \\
Spot & 62 & $2.8(5.4)$ & \\
Island & 19 & $1.3(5.2)$ & \\
Linear & & & 0.83 \\
Extension Subluxation & 7 & $3.3(3.9)$ & \\
Spot & 62 & $2.1(5.5)$ & \\
Island & 19 & $1.9(4.8)$ & \\
Linear & & & \\
\hline
\end{tabular}


Table 2

Subluxation between no surgery and surgery

\begin{tabular}{|c|c|c|c|c|}
\hline \multirow{2}{*}{\multicolumn{2}{|c|}{ Flexion Subluxation }} & \multirow{3}{*}{$\begin{array}{l}\mathbf{N} \\
4\end{array}$} & \multirow{3}{*}{$\begin{array}{l}\text { Mean (Standard Deviation) } \\
7.1(5.7)\end{array}$} & \multirow{3}{*}{$\begin{array}{l}\text { P-value } \\
0.15\end{array}$} \\
\hline & & & & \\
\hline No Previous Surgery & Spot & & & \\
\hline & Island & 42 & $2.8(5.4)$ & \\
\hline & Linear & 13 & $1.0(5.3)$ & \\
\hline \multirow[t]{3}{*}{ Previous Surgery } & Spot & 3 & $1.8(4.0)$ & \multirow[t]{3}{*}{0.88} \\
\hline & Island & 20 & $2.9(5.4)$ & \\
\hline & Linear & 6 & $1.9(5.4)$ & \\
\hline \multicolumn{5}{|l|}{ Extension Subluxation } \\
\hline \multirow[t]{3}{*}{ No Previous Surgery } & Spot & 4 & $4.9(3.9)$ & \multirow[t]{3}{*}{0.56} \\
\hline & Island & 42 & $2.5(4.9)$ & \\
\hline & Linear & 13 & $1.9(4.6)$ & \\
\hline \multirow[t]{3}{*}{ Previous Surgery } & Spot & 3 & $1.3(3.4)$ & \multirow[t]{3}{*}{0.96} \\
\hline & Island & 20 & $1.3(6.7)$ & \\
\hline & Linear & 6 & $2.2(2.6)$ & \\
\hline
\end{tabular}

We compared the anterior to posterior disc height on flexion x-rays and found that there was a significant difference within the island group ( $p=0.007)$, but there was no significant difference within the spot group $(p=0.47)$ or linear group $(p=0.39)$. We then compared the anterior to posterior disc height on extension views and found that there was a significant difference within the island group $(p<0.0001)$ and linear group $(p=0.01)$, and the difference was approaching significance within the spot group $(p=0.08)$ (Table 3). 
Table 3

Comparison of Anterior and Posterior disc height between spot, island and linear morphologies between those who have and have not had previous surgery

\begin{tabular}{|c|c|c|c|c|c|c|c|}
\hline & & \multicolumn{3}{|c|}{ Anterior Disc Height } & \multicolumn{3}{|c|}{ Posterior Disc Height } \\
\hline & & \multicolumn{2}{|c|}{ Flexion } & \multirow{2}{*}{$\begin{array}{l}\text { Extension } \\
\text { Mean (SD) }\end{array}$} & \multicolumn{2}{|c|}{ Flexion } & \multirow{2}{*}{$\begin{array}{l}\text { Extension } \\
\text { Mean (SD) }\end{array}$} \\
\hline & & $\mathbf{N}$ & Mean (SD) & & $\mathbf{N}$ & Mean (SD) & \\
\hline \multirow[t]{4}{*}{ No Previous Surgery } & Spot & 4 & $7.5(4.1)$ & $9.9(7.4)$ & 4 & $7.1(4.6)$ & $5.6(3.3)$ \\
\hline & Island & 12 & $8.5(5.3)$ & $9.9(6.1)$ & 42 & $6.1(3.6)$ & $5.8(3.5)$ \\
\hline & Linear & 13 & $5.7(3.2)$ & $7.0(4.2)$ & 13 & $4.3(3.1)$ & $3.9(2.1)$ \\
\hline & Total & 59 & & & 59 & & \\
\hline \multirow[t]{4}{*}{ Previous Surgery } & Spot & 3 & $9.9(3.9)$ & $10.6(4.9)$ & 3 & $7.1(1.7)$ & $5.5(1.5)$ \\
\hline & Island & 20 & $10.0(6.1)$ & $9.3(4.4)$ & 20 & $7.8(3.1)$ & $6.2(3.1)$ \\
\hline & Linear & 6 & $8.4(4.6)$ & $9.3(4.4)$ & 6 & $7.7(4.5)$ & $7.0(3.3)$ \\
\hline & Total & 29 & & & 29 & & \\
\hline
\end{tabular}

On sagittal CT imaging, there is a significant difference in spot VP size between no surgery and previous surgery groups $(p=0.05$; no surgery mean $=1.6, S D=0.2$, previous surgery mean $=1.1, S D=0.3)$. There is also a significant difference in linear VP size between no surgery and previous surgery groups $(p=0.008$; no surgery mean $=15.6 \mathrm{SD}=8.5$, previous surgery mean $=5.7, \mathrm{SD}=2.0)$. No significant difference was found in island VP size between no surgery and previous surgery groups $(p=0.49$; no surgery mean = $17.9, \mathrm{SD}=8.4$, previous surgery mean $=16.3 . \mathrm{SD}=10.2$ ).

\section{Discussion}

Chronic lower back pain is a common cause of disability and missed work. Over the past three decades, epidemiological studies have shown that it is the leading cause of years lived in the US with disability, and it is a tremendous economic burden on society. It affects up to $84 \%$ of the adult population. ${ }^{11}$ Lower back pain can stem from different etiologies including muscular, ligamentous, or neurologic pain, as well as the more difficult to diagnose discogenic or facetogenic pains. Unless there are red flags such as fevers, chills, increased age, a cancer diagnosis, or focal neurologic symptoms, no imaging is recommended for lower back pain for at least 6 weeks.

When conservative treatment has failed, or imaging is recommended, MRI is the imaging modality of choice. ${ }^{12}$ Neurologic symptoms may stem from causes such as disc herniations or spinal stenosis from 
bony or ligamentous hypertrophy. Once a clear radiographic finding correlating with symptoms is established, and all conservative measures including pain medications, physical therapy, and steroid injections have been exhausted, the patient may decide to pursue surgical options, such as a discectomy or spinal decompression surgery. At this point, surgeons often use CT imaging to better understand the bony anatomy to help them prepare for surgery. ${ }^{13}$ Traditionally, lateral flexion-extension radiographs are also utilized to determine if any anterior-posterior dynamic subluxation of adjacent vertebrae is present. ${ }^{14}$ If so, this may indicate vertebral instability and potentially necessitates the need for a spinal fusion with rods and screws or interbody devices in addition to spinal decompression (i.e. laminectomy).

With lower back pain causing such a societal burden, it is important to continue to discover the nuances of spinal radiology and surgery in order to refine the diagnosis and treatment of lumbar spine disorders. Recently, there has been much interest in finding validated radiographic indexes or direct measures to better measure vertebral instability pre-operatively. Examples of this include using facet joint effusion on MRI to determine translational or rotational instability. Another potential method involves using the Quantitative Stability Index based on MRI facet- fluid and flexion-extension radiographs. Still, even more potential methods exist, such as defining the Neutral Zone, which is the magnitude of residual displacement after loading and unloading a vertebral segment in a certain direction and waiting 30 seconds. ${ }^{15-17}$ In this same vein, we have looked at the presence of VP in the lumbar spine as a potential indicator of vertebral instability.

VP was first reported within IVD's by Magnusson in $1937 .{ }^{18}$ In 1942, Knuttson stated that VP was due to disc degeneration. ${ }^{19}$ The gaseous make-up within the discs was analyzed by Ford et al. in 1977 and determined to be approximately $92 \%$ nitrogen, along with oxygen, carbon dioxide, and other trace gases. ${ }^{7,9}$ VP resurfaced in literature in the 1980's and was thought to be caused by multiple etiologies including trauma, infection, malignancy, and degenerative changes, ${ }^{4}$ but multiple studies have demonstrated that its presence is largely due to end stage degenerative changes. ${ }^{20}$ Its presence in literature has been increasing in recent years, and it may be more than simply an incidental and anecdotal finding.

Past studies have looked for a correlation between IVD VP presence and vertebral instability. Lin et al. focused on surgical outcomes in patients with same segment spondylolisthesis and IVD VP presence. 8 They compared two different types of spondylolisthesis, isthmic and degenerative (IS) and (DS). While there was a larger presence of VP in IS patients compared to DS patients, the fusion rate post-operatively was also higher in IS patients. Therefore, this study concluded that IVD VP presence was not directly correlated with instability. In another study, Liao et al. also studied surgical outcomes in same segment spondylolisthesis and VP. ${ }^{21}$ In their study, they treated patients surgically with posterolateral fusion either with or without the addition of an interbody device. Less translation in the patients with interbody devices led them to conclude that VP presence at the same segment of spondylolisthesis, does indicate vertebral instability. These conflicting results led us to research if certain variables, such as size and shape, of VP may play a role in instability. 
A 2018 classification system designed by Murata et al. demonstrated that morphologic characteristics on sagittal CT and location on axial CT may be more closely associated with degenerative changes or disc height loss on MRI. This was the first time VP was classified on CT and may help radiologists determine the nuances of VP to gain a better understanding of when to mention VP in their readings. Morphology on CT images was separated into three categories: spot (less than $2 \mathrm{~mm}$ ), island (greater than $2 \mathrm{~mm}$ ), and linear. Larger island VP's greater than $2 \mathrm{~mm}$ were significant indicators of decreased disc height. Both island and linear VP's were significant indicators of high-grade degenerative disc changes on MRI. ${ }^{3}$ Due to the fact that most patients undergoing spine surgery have received CT imaging and lateral flexionextension $\mathrm{x}$-rays for pre-operative surgical guidance, we decided to retrospectively look for the presence of VP on sagittal CT. ${ }^{22}$ and use the Murata et al. morphologic component of the classification system of island (less than $2 \mathrm{~mm}$ ), spot (greater than $2 \mathrm{~mm}$ ), and linear morphologies to determine which morphology is correlated with increased dynamic subluxation or loss of disc height on lateral flexionextension x-rays. Furthermore, we performed these analyses between patients who have and who have not had previous lumbar surgery.

We hypothesized that the larger sized island and linear VP's would have larger amounts of subluxation and decreased ADH and PDH compared to the smaller spot VP's. After performing a statistical analysis, we in fact, did not find a significant difference in any of these parameters during a 3-way comparison of the morphologies. There may be multiple reasons for this finding. First, by strictly adhering to the categorical classification system, we did not separate within island or linear VP based on their size. For example, a $3 \mathrm{~mm}$ island VP was considered equal to a $20 \mathrm{~mm}$ island VP. A tiered classification distinguishing between smaller, medium, and larger sizes of island and linear VP may have provided more clarity. Second, while attempting to elucidate if one or two VP morphologies were more likely to cause vertebral instability, we compared the three VP groups to themselves instead of comparing each individual VP group against instability itself. In other words, studies have defined a subluxation of 2$4.5 \mathrm{~mm}$ as vertebral instability. ${ }^{23,25}$ If the subluxation measurements of each group would have been compared to these parameters, they very well could have indicated instability. Our overall goal was to determine if certain morphologies were better at determining instability, though, we failed to prove this. Third, one may find a multitude of spine literature questioning the validity of dynamic flexion-extension $\mathrm{x}$ rays in determining vertebral instability. ${ }^{17,24-26}$ We decided to use this radiographic option as it is still traditionally used by spine surgeons in determining instability pre-operatively, and these x-rays are usually available in the radiographic records of most patients with a history of a spine CT. Lastly, our study may have lacked sufficient power due to sample size. A larger retrospective study may have allowed the statistical analysis to reach significance when comparing between the three groups.

An interesting finding from this study is that there was a significant difference between the VP size of spot and linear morphology between patients who had never had lumbar fusion surgery and those who had a lumbar fusion surgery. This is further evidence that VP is associated with degenerative changes because decompressive surgery decreased the size of VP. Furthermore, fusion and the improvement in segmental stability leading to a decreased VP size may indicate a correlation between VP size and 
instability. Island VP size was not significantly decreased following fusion, and this may, again, be due to the wide spectrum of sizes assigned to the island classification under this CT classification system. Further dissection between small, medium, and large sized circular-shaped VP may provide a more helpful classification system when determining vertebral instability.

Another interesting finding from this study was that there was a significant difference between ADH and $\mathrm{PDH}$ on flexion and extension island VP $\mathrm{x}$-rays, and extension linear $\mathrm{x}$-rays, while there was no difference between $A D H$ and PDH on any spot $x$-rays. This may suggest that increased VP size may lead to less uniform discs, again making the case for VP and disc degeneration and potential instability. Further studies on this subject with the addition of MRI grading of disc degeneration is recommended.

Limitations to our study include the lack of a validated gold-standard metric for determining vertebral instability. This may limit the generalizability of our study, but it does not limit the within-study comparative studies. Furthermore, measurements were taken by individuals of different levels of training ( 2 medical students, 1 orthopedic surgery resident, and 1 neuroradiologist) and intra- and inter-observer reliability were not statistically analyzed. Although, intra-and inter- observer reliability were found to have sufficient kappa values in the Murata et al. study with two orthopedic surgeons. Our study only analyzed 1 part of the 2-part Murata classification system by looking strictly at VP size and morphology and neglecting the location within the IVD on axial CT images. This limited our CT readings to a 2-

dimensional analysis, whereas the Murata et al. classification system was 3-dimensional. This was done intentionally as we hoped to focus on one aspect of the classification system in determining its utility, and to potentially see how it may be improved upon. Another consideration that would allow future studies to expand on our research is to factor in the surgical outcomes as the Lin et al. and Liao et al. studies mentioned earlier did. This may factor in possible confounding variables that may be present in our study. For instance, the Lin et al. study determined that not only did IS patients have more VP, but that they had less dynamic subluxation and higher fusion rates than DS patients. This may be due to IS and DS resulting from different mechanisms. Our study also did not factor in the age, sex, or comorbid conditions of the patients. Lastly, as mentioned previously, our statistics may have been affected by a lack of power due to insufficient sample size. Still, this retrospective study provided a follow-up to the Murata et al. CT classification paper, and our data highlights potential improvements that may be made to the morphologic portion of the system.

\section{Conclusion}

Given the finding of no differences in morphology by group, we suggest that fine-tuning of this portion of the classification system may improve its diagnostic and prognostic abilities. Also, the significant difference observed between anterior and posterior disc heights on flexion-extension x-rays for island and linear VPs suggests that larger VPs may indicate degenerative changes and potential instability in agreement with recent VP literature. We recommend further research into the development of an allencompassing CT classification system for IVD VP. 


\section{Abbreviations}

IVD: Intervertebral Disc

AF: Annulus Fibrosis

NP: Nucleus Propulsus

VP: Vacuum Phenomenon

ADH: Anterior Disk Height

PDH: Posterior Disk Height

IS: Isthmic Spondylolisthesis

DS: Degenerative Spondylolisthesis

\section{Declarations}

\section{Ethics approval and consent to participate}

Ethics approval was obtained. Approval was provided by the Ascension Genesys Hospital Institutional Review Board.

\section{Consent for publication}

No individual identifiable data was used, therefore no consent for publication is required.

\section{Availability of data and materials}

The data analyzed in this study are not publicly available due to privacy and confidentiality restrictions defined by HIPAA. Deidentified data can be made available from the corresponding author on reasonable request.

\section{Competing interests}

There are no financial or non-financial competing interests to declare.

\section{Funding}

There are no funding sources to report. 


\section{Authors' contributions}

NK and MA contributed to design of the work. NK, MA, JW and EB contributed to acquisition of the data. $\mathrm{JH}$ and NK contributed to the analysis. $\mathrm{JH}, \mathrm{NK}$ and MA contributed to the interpretation of the data. NK drafted the manuscript. JH and NK contributed to revisions and editing to the manuscript.

\section{Acknowledgements}

There are no acknowledgements.

\section{References}

1. An K-C, Kong G-M, Park D-H, Baik J-M, Youn J-H, Lee W-S. Comparison of posterior lumbar interbody fusion and posterolateral lumbar fusion in monosegmental vacuum phenomenon within an intervertebral disc. Asian Spine J. 2016;10(1):93-8. doi:10.4184/asj.2016.10.1.93.

2. Feng SW, Chang MC, Wu HT, Yu JK, Wang ST, Liu CL. Are intravertebral vacuum phenomenon benign lesions. Eur Spine J. 2011;20(8):1341-8. doi:10.1007/s00586-011-1789-3.

3. Waxenbaum JA, Reddy V, Futterman B. Anatomy, back, intervertebral discs. In: StatPearls. Treasure Island (FL): StatPearls Publishing; 2020.

4. Murata K, Akeda K, Takegami N, Cheng K, Masuda K, Sudo A. Morphology of intervertebral disc ruptures evaluated by vacuum phenomenon using multi-detector computed tomography: association with lumbar disc degeneration and canal stenosis. BMC Musculoskelet Disord. 2018;19:164. doi:10.1186/s12891-018-2086-7.

5. Osti OL, Vernon-Roberts B, Moore R, Fraser RD. Annular tears and disc degeneration in the lumbar spine. A post-mortem study of 135 discs. J Bone Joint Surg Br. 1992;74(5):678-82. doi:10.1302/0301-620x.74b5.1388173.

6. Vernon-Roberts B, Moore RJ, Fraser RD. The natural history of age-related disc degeneration. Spine. 2007;32(25):2797-804. doi:10.1097/brs.0b013e31815b64d2.

7. Resnick D, Niwayama G, Guerra J, Vint V, Usselman J. Spinal vacuum phenomena: anatomical study and review. Radiology. 1981;139(2):341-8. doi:10.1148/radiology.139.2.7220878.

8. Coulier B. The spectrum of vacuum phenomenon and gas in spine. JBR-BTR. 2004;87(1):9-16.

9. Lin TY, Liao JC, Tsai TT, et al. The effects of anterior vacuum disc on surgical outcomes of degenerative versus spondylolytic spondylolisthesis: at a minimum two-year follow-up. BMC Musculoskelet Disord. 2014;15:329. doi:10.1186/1471-2474-15-329.

10. Ford LT, Gilula LA, Murphy WA, Gado M. Analysis of gas in vacuum lumbar disc. AJR Am J Roentgenol. 1977;128(6):1056-7. doi:10.2214/ajr.128.6.1056.

11. Pieper CC, Groetz SF, Nadal J, Schild HH, Niggemann PD. Radiographic evaluation of ventral instability in lumbar spondylolisthesis: do we need extension radiographs in routine exams? Eur 
Spine J. 2014;23(1):96-101. doi:10.1007/s00586-013-2932-0.

12. Shmagel A, Foley R, Ibrahim H. Epidemiology of chronic low back pain in US adults: data from the 2009-2010 national health and nutrition examination survey. Arthritis Care Res (Hoboken). 2016;68(11):1688-94. doi:10.1002/acr.22890.

13. $10.12688 /$ f1000research. 8105.2

Allegri M, Montella S, Salici F, et al. Mechanisms of low back pain: a guide for diagnosis and therapy. F1000Res. 2016;5:F1000 Faculty Rev-1530. Published 2016 Jun 28.

doi:10.12688/f1000research.8105.2.

14. Daemi N, Ahmadian A, Mirbagheri A, et al. Planning screw insertion trajectory in lumbar spinal fusion using pre-operative CT images. Conf Proc IEEE Eng Med Biol Soc. 2015;2015:3639-42. doi:10.1109/EMBC.2015.7319181.

15. Quinnell RC, Stockdale HR. Flexion and extension radiography of the lumbar spine: a comparison with lumbar discography. Clin Radiol. 1983;34(4):405-11. doi:10.1016/s0009-9260(83)80224-4.

16. Hipp JA, Guyer RD, Zigler JE, Ohnmeiss DD, Wharton ND. Development of a novel radiographic measure of lumbar instability and validation using the facet fluid sign. Int J Spine Surg. 2015;9:37. doi:10.14444/2037. Published 2015 Jul 17.

17. Lattig F, Fekete TF, Grob D, Kleinstück FS, Jeszenszky D, Mannion AF. Lumbar facet joint effusion in MRI: a sign of instability in degenerative spondylolisthesis? Eur Spine J. 2012;21(2):276-81. doi:10.1007/s00586-011-1993-1.

18. Smit TH, van Tunen MS, van der Veen AJ, Kingma I, van Dieën JH. Quantifying intervertebral disc mechanics: a new definition of the neutral zone. BMC Musculoskeletal Disroders. 2011;12(38). doi:10.1185/1471-2474-12-38.

19. Magnusson W. Uber die bendingungen des hervortretens der wirklichen gelenkspalte auf dem rontgenblide. Acta Radiol. 1937;18:733-41. doi:10.3109/00016923709132378.

20. Knutsson F. The vacuum phenomenon in the intervertebral disc. Acta Radiol. 1942;23(2):173-9. doi:10.3109/00016924209134912.

21. Li FC, Zhang N, Chen WS, Chen QX. Endplate degeneration may be the origination of the vacuum phenomenon in intervertebral discs. Med Hypotheses. 2010;75(2):169-71. doi:10.1016/j.mehy.2010.02.012.

22. Liao JC, Lu ML, Niu CC, Chen WJ, Chen LH. Surgical outcomes of degenerative lumbar spondylolisthesis with anterior vacuum disc: can the intervertebral cage overcome intradiscal vacuum phenomenon and enhance posterolateral fusion? J Orthop Sci. 2014;19(6):951-859. doi:10.1007/s00776-014-0618-z.

23. Grenier N, Grossman RI, Schiebler ML, Yeager BA, Goldberg HI, Kressel HY. Degenerative lumbar disk disease: pitfalls and usefulness of MR imaging in detection of vacuum phenomenon. Radiology. 1987;164(3):861-5. doi:10.1148/radiology.164.3.3615888.

24. Rathod AK, Dhake RP. Radiographic Incidence of Lumbar Spinal Instability in Patients with Nonspondylolisthetic Low Backache. Cureus. 2018;10(4):e2420. doi:10.7759/cureus.2420. 
25. Hayes MA, Howard TC, Gruel CR, Kopta JA. Roentgenographic evaluation of lumbar spine flexionextension in asymptomatic individuals. Spine (Phila Pa 1976). 1989;14(3):327-31. doi:10.1097/00007632-198903000-00014.

26. Hammouri QM, Haims AH, Simpson AK, Alqaqa A, Grauer JN. The utility of dynamic flexion-extension radiographs in the initial evaluation of the degenerative lumbar spine. Spine (Phila Pa 1976). 2007;32(21):2361-4. doi:10.1097/BRS.0b013e318155796e.

\section{Figures}

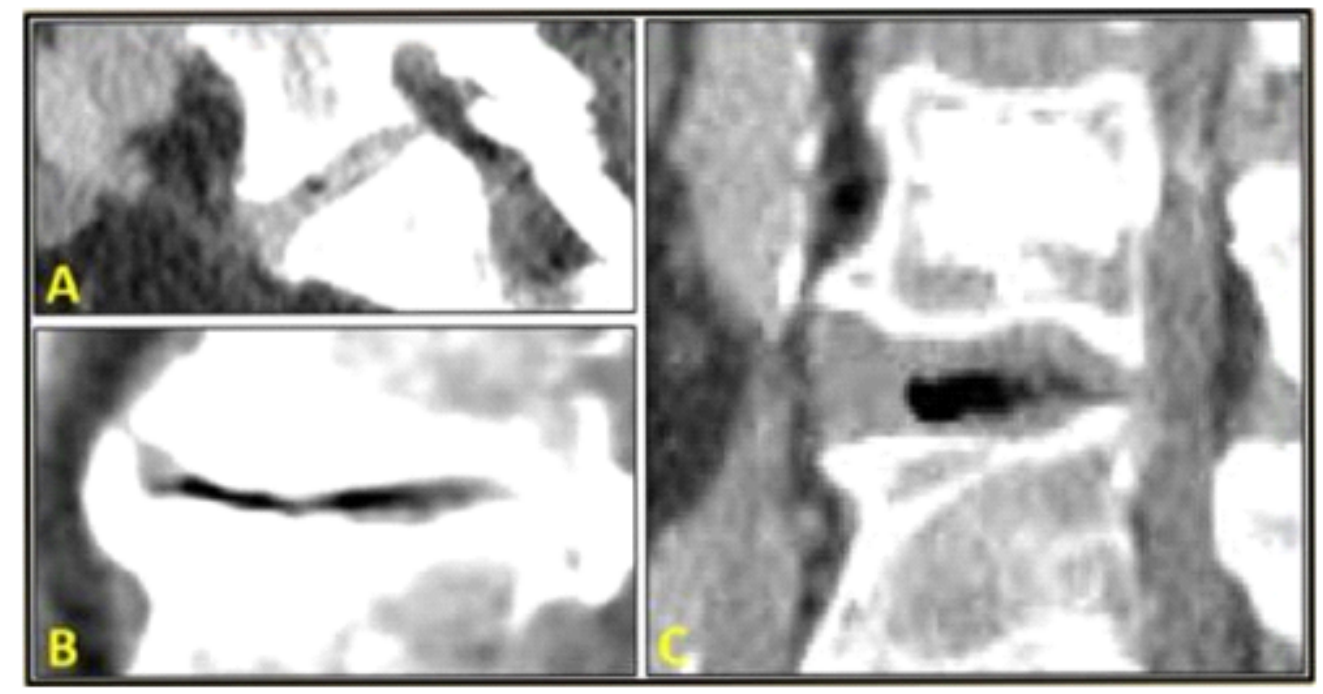

\section{Figure 1}

VP Morphology: Image (A) depicts Spot type VP (spot was considered any circular or oval-shaped VP less than $2 \mathrm{~mm}$ in diameter). Image (B) depicts Linear type VP (Linear VP was linear, serpentine, or geographical shaped VP). Image (C) depicts Island type VP (island was circular or oval and greater than 2 $\mathrm{mm}$ in diameter). 


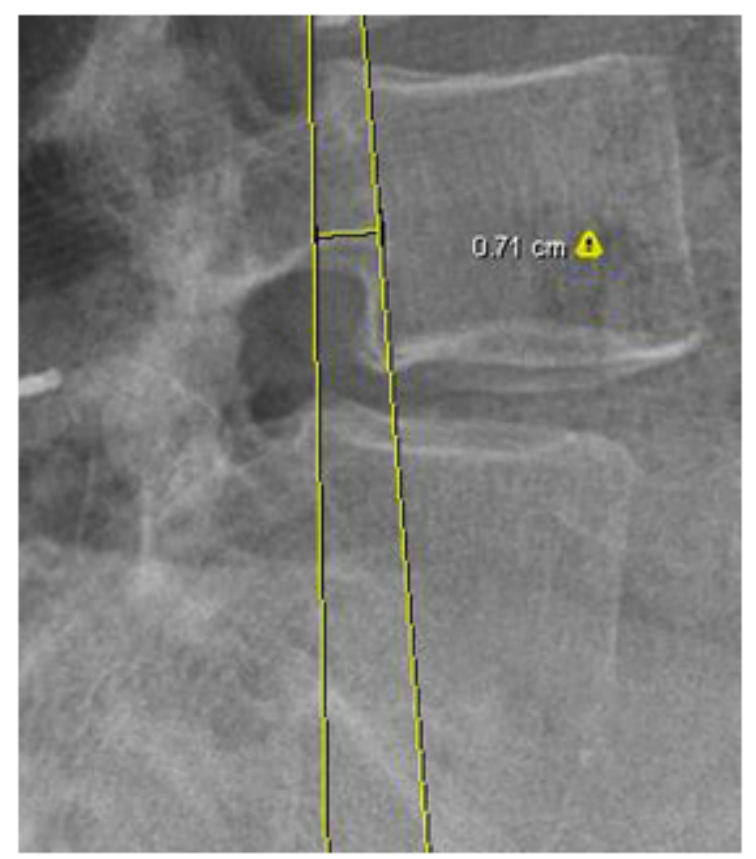

\section{Figure 2}

Subluxation Measurement: Dynamic anterior subluxation was measured on lateral $x$-rays from the midbody posterior cortex of the cephalad vertebrae to the midbody posterior cortex of the adjacent caudal vertebrae. 